\title{
On Lorentz mixed normed modulation spaces
}

\author{
Ayşe Sandıkçı
}

Received: 25 May 2012 / Accepted: 15 June 2012 / Published online: 5 July 2012

(C) The Author(s) 2012. This article is published with open access at Springerlink.com

\begin{abstract}
This paper is a study on a new kind modulation spaces $M(P, Q)\left(\mathbb{R}^{d}\right)$ and $A(P, Q, r)\left(\mathbb{R}^{d}\right)$ for indices in the range $1<P<\infty, 1 \leq Q<\infty$ and $1 \leq r<$ $\infty$, modelled on Lorentz mixed norm spaces instead of mixed norm $L^{P}$ spaces as the spaces $M_{m}^{p, q}\left(\mathbb{R}^{d}\right)$ (Feichtinger in Modulation spaces on locally compact Abelian groups, 1983; Gröchenig in Foundations of Time-Frequency Analysis. Birkh äuser, Boston, 2001), and Lorentz spaces as the spaces $M(p, q)\left(\mathbb{R}^{d}\right)$ (Gürkanliin J Math Kyoto Univ 46:595-616, 2006). First, we prove the main properties of these spaces. Later, we describe the dual spaces and determine the multiplier spaces for both of them. Moreover, we investigate the boundedness of Weyl operators and localization operators on $M(P, Q)\left(\mathbb{R}^{d}\right)$. Finally, we give an interpolation theorem for $M(P, Q)\left(\mathbb{R}^{d}\right)$.
\end{abstract}

Keywords Gabor transform · Lorentz mixed norm space · modulation space · Weyl operator $\cdot$ Multiplier

Mathematics Subject Classification $42 \mathrm{~A} 38 \cdot 43 \mathrm{~A} 15 \cdot 43 \mathrm{~A} 22 \cdot 46 \mathrm{E} 30$

\section{Introduction}

In this paper we will work on $\mathbb{R}^{d}$ with Lebesgue measure $d x$. We denote by $C_{c}\left(\mathbb{R}^{d}\right)$ and $\mathcal{S}\left(\mathbb{R}^{d}\right)$ the spaces of complex-valued continuous functions with compact support and the space of complex-valued continuous functions on $\mathbb{R}^{d}$ rapidly decreasing at infinity, respectively. Let $f$ be a complex valued measurable function on $\mathbb{R}^{d}$. The operators $T_{x} f(t)=f(t-x)$ and $M_{w} f(t)=e^{2 \pi i w t} f(t)$ are called translation and

\footnotetext{
A. Sandikçı $(\bowtie)$

Department of Mathematics, Faculty of Arts and Sciences,

University of Ondokuz Mayıs, 55139 Kurupelit, Samsun, Turkey

e-mail: ayses@omu.edu.tr
}

Birkhäuser 
modulation operator for $x, w \in \mathbb{R}^{d}$, respectively. The compositions

$$
T_{x} M_{w} f(t)=e^{2 \pi i w(t-x)} f(t-x) \text { or } M_{w} T_{x} f(t)=e^{2 \pi i w t} f(t-x)
$$

are called time-frequency shifts (see [14]). We write $\left(L^{p}\left(\mathbb{R}^{d}\right),\|\cdot\|_{p}\right)$ the Lebesgue spaces for $1 \leq p \leq \infty$.

For $f \in L^{1}\left(\mathbb{R}^{d}\right)$ the Fourier transform $\hat{f}$ (or $\mathcal{F} f$ ) is defined as

$$
\hat{f}(t)=\int_{\mathbb{R}^{d}} f(x) e^{-2 \pi i x t} d x
$$

where $x t=\sum_{i=1}^{d} x_{i} t_{i}$ is the usual scalar product on $\mathbb{R}^{d}$.

Fix a function $g \neq 0$ (called the window function). The short-time Fourier transform (STFT) of a function $f$ with respect to $g$ is given by

$$
V_{g} f(x, w)=\int_{\mathbb{R}^{d}} f(t) \overline{g(t-x)} e^{-2 \pi i t w} d t,
$$

for $x, w \in \mathbb{R}^{d}$. It is known that if $f, g \in L^{2}\left(\mathbb{R}^{d}\right)$ then $V_{g} f \in L^{2}\left(\mathbb{R}^{d} \times \mathbb{R}^{d}\right)$ and $V_{g} f$ is uniformly continuous. Moreover

$$
V_{g}\left(T_{u} M_{\eta} f\right)(x, w)=e^{-2 \pi i u w} V_{g} f(x-u, w-\eta)
$$

for all $x, w, u, \eta \in \mathbb{R}^{d}$.

The cross-Wigner distribution of $f, g \in L^{2}\left(\mathbb{R}^{d}\right)$ is defined to be

$$
W(f, g)(x, w)=\int_{\mathbb{R}^{d}} f\left(x+\frac{t}{2}\right) \overline{g\left(x-\frac{t}{2}\right)} e^{-2 \pi i t w} d t
$$

If $f=g$, then $W(f, f)=W f$ is called the Wigner distribution of $f \in L^{2}\left(\mathbb{R}^{d}\right)$. Given a symbol $\sigma \in \mathcal{S}^{\prime}\left(\mathbb{R}^{2 d}\right)$, the Weyl operator $L_{\sigma}$ is defined by

$$
\left\langle L_{\sigma} f, g\right\rangle=\langle\sigma, W(g, f)\rangle
$$

for $f, g \in \mathcal{S}\left(\mathbb{R}^{d}\right)$. The mapping $\sigma \rightarrow L_{\sigma}$ is called the Weyl transform (see [14]).

The time-frequency localization operator $A_{a}^{\varphi_{1}, \varphi_{2}}$ with symbol $a \in \mathcal{S}^{\prime}\left(\mathbb{R}^{d}\right)$ and windows $\varphi_{1}, \varphi_{2}$ is defined to be

$$
A_{a}^{\varphi_{1}, \varphi_{2}} f(t)=\int_{\mathbb{R}^{2 d}} a(x, w) V_{\varphi_{1}} f(x, w) M_{w} T_{x} \varphi_{2} d x d w
$$


Moreover, the Weyl operator and the time-frequency localization operator $A_{a}^{\varphi_{1}, \varphi_{2}}$ are related by the formula

$$
A_{a}^{\varphi_{1}, \varphi_{2}}=L_{a * W\left(\varphi_{2}, \varphi_{1}\right)},
$$

where $\sigma=a * W\left(\varphi_{2}, \varphi_{1}\right)$ (see [8]).

A weight function $w$ on $\mathbb{R}^{d}$ is a non-negative, continuous and locally integrable function. The weight $v$ is called submultiplicative if $v(x+y) \leq v(x) v(y)$ for all $x, y \in \mathbb{R}^{d}$. Let $v$ be a submultiplicative function on $\mathbb{R}^{d}$. A weight function $w$ on $\mathbb{R}^{d}$ is $v$-moderate if $w(x+y) \leq C v(x) w(y)$ for all $x, y \in \mathbb{R}^{d}$. Further, $w$ is a weight of polynomial growth if

$$
w(x) \leq C v_{s}(x)=C\left(1+|x|^{2}\right)^{\frac{s}{2}}
$$

for some $C>0, s \geq 0$ and $x \in \mathbb{R}^{d}$.

Fix a non-zero window $g \in \mathcal{S}\left(\mathbb{R}^{d}\right)$ and $1 \leq p, q \leq \infty$. Let $m$ be a weight function of polynomial growth and $v_{s}$-moderate on $\mathbb{R}^{2 d}$. Then the modulation space $M_{m}^{p, q}\left(\mathbb{R}^{d}\right)$ consists of all tempered distributions $f \in \mathcal{S}^{\prime}\left(\mathbb{R}^{d}\right)$ such that the shorttime Fourier transform $V_{g} f$ is in the weighted mixed-norm space $L_{m}^{p, q}\left(\mathbb{R}^{2 d}\right)$. The norm on $M_{m}^{p, q}\left(\mathbb{R}^{d}\right)$ is $\|f\|_{M_{m}^{p, q}}=\left\|V_{g} f\right\|_{L_{m}^{p, q}}$. If $p=q$, then we write $M_{m}^{p}\left(\mathbb{R}^{d}\right)$ instead of $M_{m}^{p, p}\left(\mathbb{R}^{d}\right)$ and if $m=1$, we have the standard modulation space $M^{p, q}\left(\mathbb{R}^{d}\right)$ (see[11,14]).

$L(p, q)$ spaces are function spaces which are closely related to $L^{p}$ spaces. We consider complex valued measurable functions $f$ defined on a measure space $(X, \mu)$. The measure $\mu$ is assumed to be nonnegative. We assume the functions $f$ are finite valued a.e. and some $y>0, \mu\left(E_{y}\right)<\infty$, where $E_{y}=E_{y}[f]=\{x \in X|| f(x) \mid>y\}$. Then, for $y>0$,

$$
\lambda_{f}(y)=\mu\left(E_{y}\right)=\mu(\{x \in X|| f(x) \mid>y\})
$$

is the distribution function of $f$. The rearrangement of $f$ is given by

$$
f^{*}(t)=\inf \left\{y>0 \mid \lambda_{f}(y) \leq t\right\}=\sup \left\{y>0 \mid \lambda_{f}(y)>t\right\}
$$

for $t>0$. The average function of $f$ is also defined by

$$
f^{* *}(x)=\frac{1}{x} \int_{0}^{x} f^{*}(t) d t .
$$

Note that $\lambda_{f}, f^{*}$ and $f^{* *}$ are nonincreasing and right continuous functions on $(0, \infty)$. If $\lambda_{f}(y)$ is continuous and strictly decreasing $f^{*}(t)$ is the inverse function of $\lambda_{f}(y)$. The most important property of $f^{*}$ is that it has the same distribution function as $f$. It follows that 


$$
\left(\int_{X}|f(x)|^{p} d \mu(x)\right)^{\frac{1}{p}}=\left(\int_{0}^{\infty}\left[f^{*}(t)\right]^{p} d t\right)^{\frac{1}{p}}
$$

The Lorentz space denoted by $L(p, q)(X, \mu)$ (shortly $L(p, q))$ is defined to be vector space of all (equivalence classes) of measurable functions $f$ such that $\|f\|_{p q}^{*}<\infty$, where

$$
\begin{aligned}
& \|f\|_{p q}^{*}=\left(\frac{q}{p} \int_{0}^{\infty} t^{\frac{q}{p}-1}\left[f^{*}(t)\right]^{q} d t\right)^{\frac{1}{q}}, \quad 0<p, q<\infty \\
& \|f\|_{p q}^{*}=\sup _{t>0} t^{\frac{1}{p}} f^{*}(t), \quad 0<p \leq q=\infty .
\end{aligned}
$$

By (1.1), it follows that $\|f\|_{p p}^{*}=\|f\|_{p}$ and so $L(p, p)=L^{p}$. Also $L(p, q)(X, \mu)$ is a normed space with the norm

$$
\begin{aligned}
& \|f\|_{p q}=\left(\frac{q}{p} \int_{0}^{\infty} t^{\frac{q}{p}-1}\left[f^{* *}(t)\right]^{q} d t\right)^{\frac{1}{q}}, \quad 0<p, q<\infty \\
& \|f\|_{p q}=\sup _{t>0}^{\frac{1}{p}} f^{* *}(t), \quad 0<p \leq q=\infty .
\end{aligned}
$$

For any one of the cases $p=q=1 ; p=q=\infty$ or $1<p<\infty$ and $1 \leq q \leq \infty$, then the Lorentz space $L(p, q)(X, \mu)$ is a Banach space with respect to the norm $\|\cdot\|_{p q}$. It is also known that if $1<p<\infty, 1 \leq q \leq \infty$ we have

$$
\|\cdot\|_{p q}^{*} \leq\|\cdot\|_{p q} \leq \frac{p}{p-1}\|\cdot\|_{p q}^{*}
$$

(see $[7,17,21])$.

It is known that by [17], $L(\infty, q)=\{0\}$ if $q \neq \infty$ and $L(\infty, q)=L^{\infty}$ if $q=\infty$. But in [1,3], $L(\infty, q)$ are defined as the class of all measurable functions $f$ for which $f^{*}(t)<\infty$ for all $t>0$ and for which $f^{* *}(t)-f^{*}(t)$ is a bounded function of $t$ such that

$$
\|f\|_{\infty q}=\left(\int_{0}^{\infty}\left[f^{* *}(t)-f^{*}(t)\right]^{q} \frac{d t}{t}\right)^{\frac{1}{q}}<\infty, \quad 0<q<\infty .
$$

Moreover, if $q=1, L(\infty, 1)=L^{\infty}$ and the norms coincide.

Let $X$ and $Y$ be two measure spaces with $\sigma$-finite measures $\mu$ and $\nu$, respectively, $f$ be a complex valued measurable function on $(X \times Y, \mu \times v), 1<P=$ $\left(p_{1}, p_{2}\right)<\infty$ and $1 \leq Q=\left(q_{1}, q_{2}\right) \leq \infty$. The Lorentz mixed norm space $L(P, Q)=L(P, Q)(X \times Y)$ is defined by 


$$
\begin{aligned}
L(P, Q)=L\left(p_{2}, q_{2}\right)\left[L\left(p_{1}, q_{1}\right)\right] & =\left\{f:\|f\|_{P Q}=\|f\|_{L\left(p_{2}, q_{2}\right)\left(L\left(p_{1}, q_{1}\right)\right)}\right. \\
& \left.=\|\| f\left\|_{p_{1} q_{1}}\right\|_{p_{2} q_{2}}<\infty\right\} .
\end{aligned}
$$

So, $L(P, Q)$ occurs by taking an $L\left(p_{1}, q_{1}\right)$-norm with respect to first variable and an $L\left(p_{2}, q_{2}\right)$-norm with respect to second variable. The $L(P, Q)$ space is a Banach space under the norm $\|\cdot\|_{P Q}($ see $[6,13])$.

Fix a window function $g \in \mathcal{S}\left(\mathbb{R}^{d}\right) \backslash\{0\}$ and $1 \leq p, q \leq \infty$. We let $M(p, q)\left(\mathbb{R}^{d}\right)$ denote the subspace of tempered distributions $\mathcal{S}^{\prime}\left(\mathbb{R}^{d}\right)$ consisting of $f \in \mathcal{S}^{\prime}\left(\mathbb{R}^{d}\right)$ such that the Gabor transform $V_{g} f$ of $f$ is in the Lorentz space $L(p, q)\left(\mathbb{R}^{2 d}\right)$. We endow it with the norm $\|f\|_{M(p, q)}=\left\|V_{g} f\right\|_{p q}$, where $\|.\|_{p q}$ is the norm of the Lorentz space. It is known that $M(p, q)\left(\mathbb{R}^{d}\right)$ is a Banach space and different windows yield equivalent norms. If $p=q$, then we denote it by $M(p, p)\left(\mathbb{R}^{d}\right)=M(p)\left(\mathbb{R}^{d}\right)$. Observe that the space $M(p)$ coincides with the standard modulation space $M^{p}$. This space $M(p, q)\left(\mathbb{R}^{d}\right)$ is defined and studied in [16]. Furthermore, the space $M(p, q)\left(\mathbb{R}^{d}\right)$ was generalized to $M(p, q, w)\left(\mathbb{R}^{d}\right)$ by taking weighted Lorentz space rather than Lorentz space in [24].

In this paper, we will denote the mixed norm space by $L^{p, q}$; the Lorentz space by $L(p, q)$; the Lorentz mixed norm space $L(P, Q)$ or sometimes $L\left(p_{2}, q_{2}\right)\left[L\left(p_{1}, q_{1}\right)\right]$, where $P=\left(p_{1}, p_{2}\right)$ and $Q=\left(q_{1}, q_{2}\right)$; the standard modulation space by $M^{p, q}$ and the modulation space which is defined using Lorentz space by $M(p, q)$.

Let $A$ be a Banach algebra and $V, W$ be left (right) Banach $A$-modules. We write $M_{A}(V, W)$ or $\operatorname{Hom}_{A}(V, W)$ for the space of all bounded linear operators satisfying $T(a b)=a T(b)$ for all $a \in A, b \in V$. This operators are called multiplier (left) or module homomorphism from $V$ into $W$ (see [22,23]).

In this paper, we introduce new generalizations of modulation spaces, called $M(P, Q)\left(\mathbb{R}^{d}\right)$ and $A(P, Q, r)\left(\mathbb{R}^{d}\right)$ spaces, which contain as special cases both the standard modulation spaces $M^{p, q}\left(\mathbb{R}^{d}\right)$, introduced by Feichtinger in 1983 , and the Lorentz-type modulation spaces $M(p, q)\left(\mathbb{R}^{d}\right)$, introduced by Gürkanlı in 2006 . We prove many properties of these spaces, in particular, they are Banach spaces, their definition is independent on the choice of the window function, convolution relations, duality relations.

Then, we study the action of Weyl operators and localization operators on the Lorentz mixed normed modulation space $M(P, Q)\left(\mathbb{R}^{d}\right)$. In particular, boundedness results are obtained, using estimates on the cross-Wigner distribution. Finally, we focus on multiplier spaces of $M(P, Q)\left(\mathbb{R}^{d}\right)$ and $A(P, Q, r)\left(\mathbb{R}^{d}\right)$.

\section{The Lorentz mixed normed modulation spaces}

\subsection{Definition and basic properties}

Throughout the paper, the letters $P$ and $Q$ will denote 2 -tuples $P=\left(p_{1}, p_{2}\right)$ and $Q=\left(q_{1}, q_{2}\right)$, where $p_{i}$ and $q_{i}, i=1,2$, are between 1 and $\infty$. When $i=1$, we shall write $P=p$ and $Q=q$. Moreover, $P \leq Q$ will mean $p_{i} \leq q_{i}$ for $i=1,2$. Further, we will agree that $\frac{1}{P}+\frac{1}{P^{\prime}}=1$ if and only if $\frac{1}{p_{1}}+\frac{1}{p_{1}^{\prime}}=1$ and $\frac{1}{p_{2}}+\frac{1}{p_{2}^{\prime}}=1$. 
Definition 1 Fix a non-zero window $g \in \mathcal{S}\left(\mathbb{R}^{d}\right), 1 \leq P=\left(p_{1}, p_{2}\right)<\infty$ and $1 \leq Q=\left(q_{1}, q_{2}\right) \leq \infty$. Then the space $M(P, Q)\left(\mathbb{R}^{\bar{d}}\right)\left(\right.$ or $\left.M\left(p_{1}, q_{1} ; p_{2}, q_{2}\right)\left(\mathbb{R}^{d}\right)\right)$ is the set of all tempered distributions $f \in \mathcal{S}^{\prime}\left(\mathbb{R}^{d}\right)$ such that the short-time Fourier transform $V_{g} f$ of $f$ is in the Lorentz mixed norm space $L(P, Q)\left(\mathbb{R}^{2 d}\right)$. We endow the vector space $M(P, Q)\left(\mathbb{R}^{d}\right)$ with the norm

$$
\|f\|_{M(P, Q)}=\left\|V_{g} f\right\|_{P Q}=\|\| V_{g} f(x, .)\left\|_{p_{1} q_{1}}\right\|_{p_{2} q_{2}}
$$

If $p_{1}=q_{1}=p$ and $p_{2}=q_{2}=q$, then the space $M(P, Q)\left(\mathbb{R}^{d}\right)$ is the standard modulation space $M^{p, q}\left(\mathbb{R}^{d}\right)$. Moreover, when $P=(p, p), Q=(q, q)$ and $p \neq q$, $L(p, q)\left(\mathbb{R}^{2 d}\right) \neq L(p, q)[L(p, q)]\left(\mathbb{R}^{2 d}\right)$ by [9, p. 287], and so $M(P, Q)\left(\mathbb{R}^{d}\right) \neq$ $M(p, q)\left(\mathbb{R}^{d}\right)$. But, since $L(P, Q)=L(p, q)$ for $P=p$ and $Q=q$, in this case $M(P, Q)\left(\mathbb{R}^{d}\right)=M(p, q)\left(\mathbb{R}^{d}\right)$.

Let $P=(\infty, \infty)$ and $1 \leq Q \leq \infty$. Then the mixed norm space $L(P, Q)\left(\mathbb{R}^{2 d}\right)$ is the set of all measurable functions $f$ for which distribution function of $f$ is finite and for which $f^{* *}-f^{*}$ is a bounded function such that

$$
\|f\|_{P Q}=\|\| f\left\|_{\infty q_{1}}\right\|_{\infty q_{2}}<\infty .
$$

Hence, we can define the space $M(P, Q)\left(\mathbb{R}^{d}\right)$ as the set of all $f \in \mathcal{S}^{\prime}\left(\mathbb{R}^{d}\right)$ such that

$$
\|f\|_{M(P, Q)}=\left\|V_{g} f\right\|_{P Q}=\|\| V_{g} f\left\|_{\infty q_{1}}\right\|_{\infty q_{2}}<\infty
$$

If $Q=(1,1)$, then $L(\infty, 1)[L(\infty, 1)]\left(\mathbb{R}^{2 d}\right)$ coincides with $L^{\infty}\left(\mathbb{R}^{2 d}\right)$, since $L(\infty, 1)\left(\mathbb{R}^{d}\right)$ is $L^{\infty}\left(\mathbb{R}^{d}\right)$ and $M(\infty, 1 ; \infty, 1)\left(\mathbb{R}^{d}\right)$ is the standard modulation space $M^{\infty}\left(\mathbb{R}^{d}\right)$. If $P=Q=(\infty, 1)$, then $L(1,1)[L(\infty, \infty)]\left(\mathbb{R}^{2 d}\right)$ is the mixed norm space $L^{\infty, 1}\left(\mathbb{R}^{2 d}\right)$ and $M(\infty, \infty ; 1,1)\left(\mathbb{R}^{d}\right)=M^{\infty, 1}\left(\mathbb{R}^{d}\right)$. Furthermore, if $P=p=\infty$ and $Q=q=1$, then $M(P, Q)\left(\mathbb{R}^{d}\right)=M(p, q)\left(\mathbb{R}^{d}\right)=M(\infty, 1)\left(\mathbb{R}^{d}\right)=M^{\infty}\left(\mathbb{R}^{d}\right)$.

Proposition 2 If $1 \leq P<\infty, 1 \leq Q<\infty$ then $\mathcal{S}\left(\mathbb{R}^{d}\right) \subset M(P, Q)\left(\mathbb{R}^{d}\right)$.

Proof Let $f \in \mathcal{S}\left(\mathbb{R}^{d}\right)$ and $P \leq Q$. Then we write $\|.\|_{P Q} \leq\|.\|_{P P}$ by Proposition 5.1 in [13] and we have

$$
\begin{aligned}
\|f\|_{M(P, Q)} & =\|\| V_{g} f(x, .)\left\|_{p_{1} q_{1}}\right\|_{p_{2} q_{2}} \\
& \leq\left\|\left\{\sup _{x \in \mathbb{R}^{d}}(1+|(x, w)|)^{n} V_{g} f(x, w)\right\}\right\|(1+|(x, .)|)^{-n}\left\|_{p_{1} q_{1}}\right\|_{p_{2} q_{2}} \\
& \leq\left\{\sup _{z \in \mathbb{R}^{2 d}}(1+|z|)^{n} V_{g} f(z)\right\}\left\|(1+|z|)^{-n}\right\|_{P Q} \\
& \leq\left\{\sup _{z \in \mathbb{R}^{2 d}}(1+|z|)^{n} V_{g} f(z)\right\}\left\|(1+|z|)^{-n}\right\|_{P P} \\
& =\left\{\sup _{z \in \mathbb{R}^{2 d}}(1+|z|)^{n} V_{g} f(z)\right\}\left\|(1+|z|)^{-n}\right\|_{L^{p_{1}, p_{2}}},
\end{aligned}
$$


where $z=(x, w) \in \mathbb{R}^{2 d}$. Then the right side of this expression is finite for sufficiently large $n$. If $P>Q$, we write

$$
\|f\|_{M(P, Q)} \leq\left\{\sup _{z \in \mathbb{R}^{2 d}}(1+|z|)^{n} V_{g} f(z)\right\}\|\|(1+|(x, .)|)^{-n}\left\|_{p_{1} q_{1}}\right\|_{p_{2} q_{2}} .
$$

By one variable proof [16, Lemma 2.1], $\left\|(1+|(x, .)|)^{-n}\right\|_{p_{1} q_{1}}<\infty$ for sufficiently large $n$. The proof is completed by repeating the same procedure with respect to the second variable.

Remark 3 It is known that the Lorentz space $L(p, q)\left(\mathbb{R}^{d}\right)$ is translation invariant and holds $\left\|T_{x} f\right\|_{p q}=\|f\|_{p q}$ for $x \in \mathbb{R}^{d}$ (see [7]). By using iteration and the one dimensional proofs given in [7] it can easily be shown that the Lorentz mixed norm space $L(P, Q)\left(\mathbb{R}^{2 d}\right)$ is also translation invariant and holds $\left\|T_{z} f\right\|_{P Q}=\|f\|_{P Q}$, for $z \in \mathbb{R}^{2 d}$.

The following proposition gives us convolution relations between Lorentz mixed norm space and $L^{1}$ space and will be used frequently.

Proposition 4 If $1<P<\infty, 1 \leq Q \leq \infty, F \in L^{1}\left(\mathbb{R}^{2 d}\right)$ and $G \in L(P, Q)\left(\mathbb{R}^{2 d}\right)$, then $F * G \in L(P, Q)\left(\mathbb{R}^{2 d}\right)$ and

$$
\|F * G\|_{P Q} \leq\|F\|_{1}\|G\|_{P Q} .
$$

Proof Let $H \in L\left(P^{\prime}, Q^{\prime}\right)\left(\mathbb{R}^{2 d}\right)$. By Fubini Theorem and Hölder inequality for Lorentz mixed norm space [13], we can change order of integration and obtain

$$
\begin{aligned}
|\langle F * G, H\rangle| & =\left|\int_{\mathbb{R}^{2 d}} \int_{\mathbb{R}^{2 d}} F(w) G(z-w) \overline{H(z)} d w d z\right| \\
& \leq \int_{\mathbb{R}^{2 d}}|F(w)|\left(\int_{\mathbb{R}^{2 d}}|G(z-w) \overline{H(z)}| d z\right) d w \\
& \leq \int_{\mathbb{R}^{2 d}}|F(w)|\|G(z-w)\|_{P Q}\|H\|_{P^{\prime} Q^{\prime}} d w \\
& =\int_{\mathbb{R}^{2 d}}|F(w)| d w\|G\|_{P Q}\|H\|_{P^{\prime} Q^{\prime}}=\|F\|_{1}\|G\|_{P Q}\|H\|_{P^{\prime} Q^{\prime}}
\end{aligned}
$$

Then by duality we get

$$
\|F * G\|_{P Q}=\sup _{\|H\|_{P^{\prime} Q^{\prime}} \leq 1}|\langle F * G, H\rangle| \leq\|F\|_{1}\|G\|_{P Q} .
$$


Theorem 5 Assume that $1<P<\infty, 1 \leq Q<\infty$ and $g, \gamma \in \mathcal{S}\left(\mathbb{R}^{d}\right)$ are non-zero window functions. Then

1. $V_{\gamma}^{*}$ maps $L(P, Q)\left(\mathbb{R}^{2 d}\right)$ into $M(P, Q)\left(\mathbb{R}^{d}\right)$ and satisfies

$$
\left\|V_{\gamma}^{*} F\right\|_{M(P, Q)} \leq\left\|V_{g} \gamma\right\|_{1}\|\| F\left\|_{p_{1} q_{1}}\right\|_{p_{2}, q_{2}} .
$$

2. If $F=V_{g} f$, then the inversion formula

$$
f=\frac{1}{\langle\gamma, g\rangle} \iint_{\mathbb{R}^{2 d}} V_{g} f(x, y) M_{y} T_{x} \gamma d x d y
$$

holds in $M(P, Q)\left(\mathbb{R}^{d}\right)$. In short, $I_{M(P, Q)}=\langle\gamma, g\rangle^{-1} V_{\gamma}^{*} V_{g}$.

Proof 1. Take any $F \in L(P, Q)\left(\mathbb{R}^{2 d}\right)$. We show first that $V_{\gamma}^{*} F \in \mathcal{S}^{\prime}\left(\mathbb{R}^{d}\right)$. Let $f \in \mathcal{S}\left(\mathbb{R}^{d}\right)$. As $\mathcal{S}\left(\mathbb{R}^{d}\right) \subset M\left(P^{\prime}, Q^{\prime}\right)\left(\mathbb{R}^{d}\right)$ by Proposition 2 , then, $V_{\gamma} f \in$ $L\left(P^{\prime}, Q^{\prime}\right)\left(\mathbb{R}^{2 d}\right)$, where $\frac{1}{P}+\frac{1}{P^{\prime}}=1$ and $\frac{1}{Q}+\frac{1}{Q^{\prime}}=1$. Using Hölder inequality for Lorentz mixed norm space, we write

$$
\left|\left\langle V_{\gamma}^{*} F, f\right\rangle\right|=\left|\iint_{\mathbb{R}^{2 d}} F(x, y) \overline{V_{\gamma} f(x, y)} d x d y\right| \leq\|F\|_{P Q}\left\|V_{\gamma} f\right\|_{P^{\prime} Q^{\prime}} .
$$

Now let $P \geq Q$ (hence $P^{\prime} \leq Q^{\prime}$ ). Then we have

$$
\begin{aligned}
\left|\left\langle V_{\gamma}^{*} F, f\right\rangle\right| & \leq\|F\|_{P Q}\left\|V_{\gamma} f\right\|_{P^{\prime} Q^{\prime}} \leq\|F\|_{P Q}\left\|V_{\gamma} f\right\|_{P^{\prime} P^{\prime}} \\
& \leq\|F\|_{P Q}\left\{\sup _{z \in \mathbb{R}^{2 d}}(1+|z|)^{n} V_{g} f(z)\right\}\left\|(1+|z|)^{-n}\right\|_{L^{p_{1}^{\prime}, p_{2}^{\prime}}}
\end{aligned}
$$

where $z=(x, w) \in \mathbb{R}^{2 d}$. This expression is finite for sufficiently large $n$. Using the equivalence of the seminorms (see [14, Corollary 11.2.6]), we have $V_{\gamma}^{*} F \in$ $\mathcal{S}^{\prime}\left(\mathbb{R}^{d}\right)$. If $P<Q$ (hence $P^{\prime}>Q^{\prime}$ ), then

$$
\left\|(1+|z|)^{-n}\right\|_{P^{\prime} Q^{\prime}}<\infty
$$

for sufficiently large $n$ by Lemma 2.1 in [16]. Thus

$$
\left|\left\langle V_{\gamma}^{*} F, f\right\rangle\right| \leq\|F\|_{P Q}\left\{\sup _{z \in \mathbb{R}^{2 d}}(1+|z|)^{n} V_{g} f(z)\right\}\left\|(1+|z|)^{-n}\right\|_{P^{\prime} Q^{\prime}}
$$

is finite. Hence $V_{\gamma}^{*} F \in \mathcal{S}^{\prime}\left(\mathbb{R}^{d}\right)$. Since $g \in \mathcal{S}\left(\mathbb{R}^{d}\right)$ and $V_{\gamma}^{*} F \in \mathcal{S}^{\prime}\left(\mathbb{R}^{d}\right)$, we have

$$
V_{g} V_{\gamma}^{*} F(u, t)=\iint_{\mathbb{R}^{2 d}} F(x, y) e^{-2 \pi i x(t-y)} V_{g} \gamma(u-x, t-y) d x d y
$$


and

$$
\left|V_{g} V_{\gamma}^{*} F(u, t)\right| \leq\left(|F| *\left|V_{g} \gamma\right|\right)(u, t)
$$

Since $V_{g} \gamma \in \mathcal{S}\left(\mathbb{R}^{2 d}\right) \subset L^{1}\left(\mathbb{R}^{2 d}\right)$, by Proposition 4 and (2.3), we obtain

$$
\left\|V_{\gamma}^{*} F\right\|_{M(P, Q)}=\left\|V_{g}\left(V_{\gamma}^{*} F\right)\right\|_{P Q} \leq\|F\|_{P Q}\left\|V_{g} \gamma\right\|_{1} .
$$

2. If $F=V_{g} f \in L(P, Q)\left(\mathbb{R}^{2 d}\right)$, then $\tilde{f}=\frac{1}{\langle\gamma, g\rangle} V_{\gamma}^{*} V_{g} f \in M(P, Q)\left(\mathbb{R}^{d}\right)$ by the above proof. As every element of $M(P, Q)\left(\mathbb{R}^{d}\right)$ is a tempered distribution, then $\widetilde{f}=f$ by $[14$, Corollary 11.2.7].

By using the same proof technique as that employed in Proposition 11.3.4 and Theorem 11.3.5 in [14], it is easy to prove the following two theorems.

Theorem $6 \mathcal{S}\left(\mathbb{R}^{d}\right)$ is dense in $M(P, Q)\left(\mathbb{R}^{d}\right)$ for $1<P<\infty, 1 \leq Q<\infty$.

Theorem 7 The normed space $M(P, Q)\left(\mathbb{R}^{d}\right)$ is a Banach space for $1<P<\infty, 1$ $\leq Q<\infty$. Moreover $M(P, Q)\left(\mathbb{R}^{d}\right)$ is independent of the window $g \in \mathcal{S}\left(\mathbb{R}^{d}\right) \backslash\{0\}$. Different windows yield equivalent norms.

It is known that the Lorentz spaces $L\left(p_{1}, q_{1}\right)$ and $L\left(p_{2}, q_{2}\right)$ have absolutely continuous norms when $1<p_{i}<\infty, 1 \leq q_{i}<\infty, i=1,2$ (see [4]). Then the space $L(P, Q)$ has also absolutely continuous norm and $(L(P, Q))^{*}=L\left(P^{\prime}, Q^{\prime}\right)$ for $1<P<\infty, 1 \leq Q<\infty$ (see [6, p. 158]). The next theorem gives us the dual space of $M(P, Q)\left(\mathbb{R}^{d}\right)$. The proof is the same as that of Theorem 2.3 in [16].

Theorem 8 Let $1<P<\infty$ and $1 \leq Q<\infty$. Then $\left(M(P, Q)\left(\mathbb{R}^{d}\right)\right)^{*}=$ $M\left(P^{\prime}, Q^{\prime}\right)\left(\mathbb{R}^{d}\right)$, where $\frac{1}{P}+\frac{1}{P^{\prime}}=1$ and $\frac{1}{Q}+\frac{1}{Q^{\prime}}=1$.

The inclusion property of $M(P, Q)\left(\mathbb{R}^{d}\right)$ spaces is connected with the indices $Q$ as in $L(P, Q)$ spaces. Let $Q_{1}=\left(q_{1}^{1}, q_{1}^{2}\right)$ and $Q_{2}=\left(q_{2}^{1}, q_{2}^{2}\right)$. Recall that $Q_{1} \leq Q_{2}$ if and only if $q_{1}^{i} \leq q_{2}^{i}, i=1,2$.

Proposition 9 Let $Q_{1} \leq Q_{2}$. Then $M\left(P, Q_{1}\right)\left(\mathbb{R}^{d}\right) \subset M\left(P, Q_{2}\right)\left(\mathbb{R}^{d}\right)$.

Proof Let $f \in M\left(P, Q_{1}\right)\left(\mathbb{R}^{d}\right)$. Then $V_{g} f \in L\left(P, Q_{1}\right)\left(\mathbb{R}^{2 d}\right)$. If $Q_{1} \leq Q_{2}$, then $L\left(P, Q_{1}\right)\left(\mathbb{R}^{2 d}\right) \subset L\left(P, Q_{2}\right)\left(\mathbb{R}^{2 d}\right)$ by Proposition 5.1 in [13]. Thus we have $V_{g} f \in$ $L\left(P, Q_{2}\right)\left(\mathbb{R}^{2 d}\right)$ and hence $f \in M\left(P, Q_{2}\right)\left(\mathbb{R}^{d}\right)$.

Theorem 10 The space $M(P, Q)\left(\mathbb{R}^{d}\right)$ is invariant under time-frequency shifts. Moreover, if $1<P<\infty$ and $1 \leq Q<\infty$, then the mapping $(u, \eta) \longmapsto M_{\eta} T_{u} f$, from $\mathbb{R}^{2 d}$ into $M(P, Q)\left(\mathbb{R}^{d}\right)$, is continuous.

Proof Let $f \in M(P, Q)\left(\mathbb{R}^{d}\right)$. Then $V_{g} f \in L(P, Q)\left(\mathbb{R}^{2 d}\right)$. Using the equality $T_{(u, \eta)} V_{g} f(x, y)=e^{2 \pi i(y-\eta) u} V_{g}\left(M_{\eta} T_{u} f\right)(x, y)$ and the fact that the Lorentz mixed norm space is translation invariant, we have

$$
\begin{aligned}
\left\|M_{\eta} T_{u} f\right\|_{M(P, Q)} & =\left\|V_{g}\left(M_{\eta} T_{u} f\right)\right\|_{P Q}=\left\|e^{2 \pi i(\eta-y) u} V_{g} f(x-u, w-\eta)\right\|_{P Q} \\
& =\left\|T_{(u, \eta)} V_{g} f\right\|_{P Q}=\left\|V_{g} f\right\|_{P Q}=\|f\|_{M(P, Q)} .
\end{aligned}
$$


Hence $M(P, Q)\left(\mathbb{R}^{d}\right)$ is invariant under time-frequency shifts. Now let $f \in$ $M(P, Q)\left(\mathbb{R}^{d}\right)$ and $u, \eta \in \mathbb{R}^{d}$. We write

$$
\begin{aligned}
\left\|M_{\eta} T_{u} f-f\right\|_{M(P, Q)}= & \left\|V_{g}\left(M_{\eta} T_{u} f\right)-V_{g} f\right\|_{P Q}=\left\|e^{2 \pi i(\eta-y) u} T_{(u, \eta)} V_{g} f-V_{g} f\right\|_{P Q} \\
\leq & \left\|e^{2 \pi i(\eta-y) u} T_{(u, \eta)} V_{g} f-e^{2 \pi i(\eta-y) u} V_{g} f\right\|_{P Q} \\
& +\left\|e^{2 \pi i(\eta-y) u} V_{g} f-V_{g} f\right\|_{P Q} \\
= & \left\|T_{(u, \eta)} V_{g} f-V_{g} f\right\|_{P Q}+\left\|\left(e^{2 \pi i(\eta-y) u}-1\right) V_{g} f\right\|_{P Q} \\
= & \|\| T_{(u, .)} V_{g} f-V_{g} f\left\|_{p_{1} q_{1}}\right\|_{p_{2} q_{2}} \\
& +\|\|\left(e^{2 \pi i(.-y) u}-1\right) V_{g} f\left\|_{p_{1} q_{1}}\right\|_{p_{2} q_{2} .} .
\end{aligned}
$$

Since the translation operator is continuous from $\mathbb{R}^{d}$ into $L(p, q)\left(\mathbb{R}^{d}\right)$ by Lemma 3.2 in [7], then $(u,.) \longmapsto T_{(u, .)} V_{g} f$ is continuous from $\mathbb{R}^{d}$ into $L\left(p_{1}, q_{1}\right)\left(\mathbb{R}^{d}\right)$. Repeating the same procedure with respect to the second variable we get $\| T_{(u, \eta)}$ $V_{g} f-V_{g} f \|_{P Q} \rightarrow 0$ as $(u, \eta)$ tends to zero. Moreover, it is known that $\|\left(e^{2 \pi i(.-y) u}-\right.$ 1) $V_{g} f \|_{p_{1} q_{1}}$ tends to zero as $u$ tends to zero by the proof of the Proposition 2.2 in [16]. The continuity of $\|\cdot\|_{p_{2} q_{2}}$ implies that $\left\|\left(e^{2 \pi i(\eta-y) u}-1\right) V_{g} f\right\|_{P Q} \rightarrow 0$ as $(u, \eta)$ tends to zero. This completes the proof.

\subsection{Convolution theorems}

Theorem 11 Let $1<P<\infty$ and $1 \leq Q<\infty$. If $f \in M(P, Q)\left(\mathbb{R}^{d}\right)$ and $h \in$ $L^{1}\left(\mathbb{R}^{d}\right)$, then $f * h \in M(P, Q)\left(\mathbb{R}^{d}\right)$ and satisfies

$$
\|f * h\|_{M(P, Q)} \leq\|f\|_{M(P, Q)}\|h\|_{1} .
$$

Proof Let $f \in M(P, Q)\left(\mathbb{R}^{d}\right)$ and let $h \in L^{1}\left(\mathbb{R}^{d}\right)$. Then $V_{g} f \in L(P, Q)\left(\mathbb{R}^{2 d}\right)$. By using the equality $V_{g} f(x, w)=e^{-2 \pi i x w}\left(f * M_{w} g^{\sim}\right)(x)$, where $g^{\sim}(x)=\overline{g(-x) \text {, }}$ and the fact that $L(P, Q)\left(\mathbb{R}^{2 d}\right)$ is strongly translation invariant by Remark 3, we write

$$
\begin{aligned}
\|f * h\|_{M(P, Q)} & =\left\|V_{g}(f * h)\right\|_{P Q}=\left\|h *\left(f * M_{w} g^{\sim}\right)\right\|_{P Q} \\
& =\left\|\int_{\mathbb{R}^{d}} h(t)\left(f * M_{w} g^{\sim}\right)(x-t) d t\right\|_{P Q} \\
& \leq \int_{\mathbb{R}^{d}}|h(t)|\left\|T_{(t, 0)} V_{g} f\right\|_{P Q} d t \\
& =\int_{\mathbb{R}^{d}}|h(t)|\left\|V_{g} f\right\|_{P Q} d t=\left\|V_{g} f\right\|_{P Q} \int_{\mathbb{R}^{d}}|h(t)| d t \\
& =\|f\|_{M(P, Q)}\|h\|_{1} .
\end{aligned}
$$

This completes the proof. 
Theorem 12 Let $1<P_{1}, P_{2}<\infty, 1 \leq Q_{1}, Q_{2} \leq \infty, \frac{1}{P_{1}}+\frac{1}{P_{2}}>1, f \in M\left(P_{1}, Q_{1}\right)$ $\left(\mathbb{R}^{d}\right), h \in M\left(P_{2}, Q_{2}\right)\left(\mathbb{R}^{d}\right)$, then $f * h \in M(R, S)\left(\mathbb{R}^{d}\right)$, where $\frac{1}{R}=\frac{1}{P_{1}}+\frac{1}{P_{2}}-1$ and $S>0$ is any number such that $\frac{1}{Q_{1}}+\frac{1}{Q_{2}} \geq \frac{1}{S}$, and

$$
M\left(P_{1}, Q_{1}\right)\left(\mathbb{R}^{d}\right) * M\left(P_{2}, Q_{2}\right)\left(\mathbb{R}^{d}\right) \hookrightarrow M(R, S)\left(\mathbb{R}^{d}\right)
$$

with norm inequality

$$
\|f * h\|_{M(R, S)} \leq\|f\|_{M\left(P_{1}, Q_{1}\right)}\|h\|_{M\left(P_{2}, Q_{2}\right)}
$$

where $P_{1}=\left(p_{1}^{1}, p_{1}^{2}\right), P_{2}=\left(p_{2}^{1}, p_{2}^{2}\right), Q_{1}=\left(Q_{1}^{1}, Q_{1}^{2}\right), Q_{2}=\left(Q_{2}^{1}, Q_{2}^{2}\right), R=$ $\left(r_{1}, r_{2}\right)$ and $S=\left(s_{1}, s_{2}\right)$.

Proof Let us choose the windows as in Proposition 2.4 in [8]. Namely, $g_{0}(x)=$ $e^{-\pi x^{2}}$ and $g(x)=2^{-\frac{n}{d}} e^{-\frac{\pi x^{2}}{2}}=\left(g_{0} * g_{0}\right)(x) \in \mathcal{S}\left(\mathbb{R}^{d}\right)$. Moreover, it is known that different windows yield equivalent norms for the spaces $M(R, S)\left(\mathbb{R}^{d}\right)$ by Theorem 7. Let $f \in M\left(P_{1}, Q_{1}\right)\left(\mathbb{R}^{d}\right)$ and $h \in M\left(P_{2}, Q_{2}\right)\left(\mathbb{R}^{d}\right)$. Then $V_{g_{0}} f \in$ $L\left(P_{1}, Q_{1}\right)\left(\mathbb{R}^{2 d}\right), V_{g_{0}} h \in L\left(P_{2}, Q_{2}\right)\left(\mathbb{R}^{2 d}\right)$. Using the equalities $V_{g_{0}} f(x, w)=$ $e^{-2 \pi i x w}\left(f * M_{w} g_{0}^{\tilde{\tau}}\right)(x), M_{w}\left(g_{0}^{\tilde{\tau}} * g_{0}^{\tilde{\tau}}\right)=M_{w} g_{0}^{\tilde{\tau}} * M_{w} g_{0}^{\sim}$, Minkowski integral inequality and Theorem 2.12 in [5], we write

$$
\begin{aligned}
& \left\|V_{g}(f * h)\right\|_{r_{1} s_{1}}\left(\eta_{2}\right)=\left\|e^{-2 \pi i x w}\left((f * h) * M_{w} g_{0}^{\sim}\right)\right\|_{r_{1} s_{1}}\left(\eta_{2}\right) \\
& =\left\|\left(f * M_{w} g_{0}^{\sim}\right) *\left(h * M_{w} g_{0}^{\sim}\right)\right\|_{r_{1} s_{1}}\left(\eta_{2}\right) \\
& \leq\left(\left\|f * M_{w} g_{0}^{\tilde{u}}\right\|_{p_{1}^{1} q_{1}^{1}} *\left\|h * M_{w} g_{0}^{\tilde{0}}\right\|_{p_{2}^{1} q_{2}^{1}}\right)\left(\eta_{2}\right) \\
& =\left(\left\|V_{g_{0}} f\right\|_{p_{1}^{1} q_{1}^{1}} *\left\|V_{g_{0}} h\right\|_{p_{2}^{1} q_{2}^{1}}\right)\left(\eta_{2}\right) \text {. }
\end{aligned}
$$

Applying the same procedure with respect to the second variable, we obtain

$$
\begin{aligned}
\|f * h\|_{M(R, S)} & \leq\|\| V_{g_{0}} f\left\|_{p_{1}^{1} q_{1}^{1}}\right\|_{p_{1}^{2} q_{1}^{2}}\|\| V_{g_{0}} h\left\|_{p_{2}^{1} q_{2}^{1}}\right\|_{p_{2}^{2} q_{2}^{2}} \\
& =\|f\|_{M\left(P_{1}, Q_{1}\right)}\|h\|_{M\left(P_{2}, Q_{2}\right)} .
\end{aligned}
$$

This is desired result.

Theorem 11 implies one of the conditions to be Banach module. The next theorem refers to this property and is proved using the same argument as Theorem 2.2 in [16]. Moreover, it is necessary to find the multiplier space of $M(P, Q)\left(\mathbb{R}^{d}\right)$.

Theorem 13 Let $1<P<\infty$ and $1 \leq Q<\infty$. Then $M(P, Q)\left(\mathbb{R}^{d}\right)$ is an essential Banach convolution module over $L^{1}\left(\mathbb{R}^{d}\right)$. 
2.3 Boundedness of Weyl operators and localization operators

Theorem 14 Let $\sigma \in M(\infty, \infty ; 1,1)\left(\mathbb{R}^{2 d}\right)$. If $1<P<\infty, 1 \leq Q \leq \infty$, then $L_{\sigma}$ is bounded from $M(P, Q)\left(\mathbb{R}^{d}\right)$ to $M(P, Q)\left(\mathbb{R}^{d}\right)$ with norm estimate

$$
\left\|L_{\sigma} f\right\|_{o p} \leq\|\sigma\|_{M(\infty, \infty ; 1,1)}
$$

Proof It follows from a small variation of Gröchenig's proof (see [14, Theorem 14.5.2]).

The following proposition and theorem gives us an estimate for the cross-Wigner distribution for Lorentz mixed normed modulation space and the boundedness of the time-frequency localization operator on $M(P, Q)\left(\mathbb{R}^{d}\right)$, respectively. The proofs are similar to Proposition 2.5 and Theorem 3.2 in [8] but let us provide the details anyway, for completeness' sake.

Proposition 15 Let $P=\left(1, p_{2}\right), Q=\left(q_{1}, q_{2}\right), 1 \leq Q<\infty$ and $1<p_{2}<\infty$. If $\varphi_{1} \in M^{1}\left(\mathbb{R}^{d}\right)$ and $\varphi_{2} \in M\left(p_{2}, q_{2}\right)\left(\mathbb{R}^{d}\right)$, then $W\left(\varphi_{2}, \varphi_{1}\right) \in M(P, Q)\left(\mathbb{R}^{2 d}\right)$ and satisfies

$$
\left\|W\left(\varphi_{2}, \varphi_{1}\right)\right\|_{M(P, Q)} \leq\left\|\varphi_{1}\right\|_{M^{1}}\left\|\varphi_{2}\right\|_{M\left(p_{2}, q_{2}\right)} .
$$

Proof Let $\varphi_{1}, \varphi_{2}, g \in \mathcal{S}\left(\mathbb{R}^{d}\right)$. Then $W\left(\varphi_{2}, \varphi_{1}\right) \in \mathcal{S}\left(\mathbb{R}^{2 d}\right)$ and so $V_{\Phi}\left(W\left(\varphi_{2}, \varphi_{1}\right)\right) \in$ $\mathcal{S}\left(\mathbb{R}^{4 d}\right)$ by Lemma 14.5.1(a) and Theorem 11.2.5 in [14], where $\Phi=W g \in \mathcal{S}\left(\mathbb{R}^{2 d}\right)$. Moreover, it is known that

$$
\left|\mathcal{V}_{\Phi} W\left(\varphi_{2}, \varphi_{1}\right)(z, \zeta)\right|=\left|V_{g} \varphi_{1}\left(z+\frac{\tilde{\zeta}}{2}\right)\right|\left|V_{g} \varphi_{2}\left(z-\frac{\tilde{\zeta}}{2}\right)\right|
$$

by Lemma 14.5.1.b. in [14], where $\zeta=\left(\zeta_{1}, \zeta_{2}\right) \in \mathbb{R}^{2 d}, \tilde{\zeta}=\left(\zeta_{2},-\zeta_{1}\right)$. On the other hand, if $\varphi_{1} \in \mathcal{S}\left(\mathbb{R}^{d}\right)$, then it is known that $\varphi_{1}$ is in the standard modulation space $M^{1}\left(\mathbb{R}^{d}\right)$, if $\varphi_{2} \in \mathcal{S}\left(\mathbb{R}^{d}\right)$, then $\varphi_{2} \in M\left(p_{2}, q_{2}\right)\left(\mathbb{R}^{d}\right)$ by Proposition 2.1 in [16]. Thus by using the inequality $\|\cdot\|_{1 q_{1}} \leq\|\cdot\|_{11}=\|\cdot\|_{1}$ when $1 \leq q_{1}$ and changing variables $z \rightarrow z-\frac{\tilde{\zeta}}{2}$, we have

$$
\begin{aligned}
\left\|\mathcal{V}_{\Phi} W\left(\varphi_{2}, \varphi_{1}\right)\right\|_{1 q_{1}}(\zeta) & \leq\left\|\mathcal{V}_{\Phi} W\left(\varphi_{2}, \varphi_{1}\right)\right\|_{1}(\zeta) \\
& =\left|V_{g} \varphi_{1}\right| *\left|V_{g} \varphi_{2}\right|(\tilde{\zeta})
\end{aligned}
$$

Again using the fact that the Lorentz space $L\left(p_{2}, q_{2}\right)\left(\mathbb{R}^{2 d}\right)$ is an essential Banach convolution module over $L^{1}\left(\mathbb{R}^{2 d}\right)$, we obtain

$$
\begin{aligned}
\left\|W\left(\varphi_{2}, \varphi_{1}\right)\right\|_{M(P, Q)} & =\|\| \mathcal{V}_{\Phi} W\left(\varphi_{2}, \varphi_{1}\right)\left\|_{1 q_{1}}\right\|_{p_{2} q_{2}} \\
& \leq\left\|\left|V_{g} \varphi_{1}\right| *\left|V_{g} \varphi_{2}\right|\right\|_{p_{2} q_{2}} \leq\left\|V_{g} \varphi_{1}\right\|_{1}\left\|V_{g} \varphi_{2}\right\|_{p_{2} q_{2}} \\
& =\left\|\varphi_{1}\right\|_{M^{1}}\left\|\varphi_{2}\right\|_{p_{2} q_{2}} .
\end{aligned}
$$


Theorem 16 Let $1<P<\infty, 1 \leq Q \leq \infty, a \in M^{\infty}\left(\mathbb{R}^{2 d}\right), \varphi_{1}, \varphi_{2} \in M^{1}\left(\mathbb{R}^{d}\right)$. Then the localization operator $A_{a}^{\varphi_{1}, \varphi_{2}}$ is bounded on $M(P, Q)\left(\mathbb{R}^{d}\right)$. Moreover we have the norm estimate

$$
\left\|A_{a}^{\varphi_{1}, \varphi_{2}}\right\|_{o p} \leq\|a\|_{M^{\infty}}\left\|\varphi_{1}\right\|_{M^{1}}\left\|\varphi_{2}\right\|_{M^{1}}
$$

Proof Let $\varphi_{1}, \varphi_{2} \in M^{1}\left(\mathbb{R}^{d}\right)$. Then $W\left(\varphi_{2}, \varphi_{1}\right) \in M^{1}\left(\mathbb{R}^{d}\right)$ and $\sigma=a * W\left(\varphi_{2}, \varphi_{1}\right) \in$ $M^{\infty, 1}\left(\mathbb{R}^{2 d}\right)$ by Proposition 2.5 and convolution relations for the standard modulation space in [8], respectively. Thus the operator $A_{a}^{\varphi_{1}, \varphi_{2}}=L_{a * W\left(\varphi_{2}, \varphi_{1}\right)}$ is bounded on $M(P, Q)\left(\mathbb{R}^{d}\right)$ for all $1<P<\infty, 1 \leq Q \leq \infty$ from Theorem 14 .

We observe that this result extends Theorem 3.2 in [8], since the same sufficient conditions provide boundedness on both classical and Lorentz mixed normed modulation spaces.

\subsection{Interpolation theorems}

Now we will give interpolation theorems for the $M(P, Q)\left(\mathbb{R}^{d}\right)$ spaces. The interpolation theorems for Lorentz mixed norm spaces are given in [20, p. 6] suggests a similar statement for the $M(P, Q)\left(\mathbb{R}^{d}\right)$ spaces. So we omit the proof.

Proposition 17 Let $0<\theta<1, \frac{1}{r_{i}}=\frac{1-\theta}{p_{i}}+\frac{\theta}{q_{i}}, \frac{1}{u_{i}}=\frac{1-\theta}{s_{i}}+\frac{\theta}{t_{i}}, i=1,2$. Let $\mathbf{T}$ be a linear operator mapping $M^{p_{1}, p_{2}}$ into $M\left(s_{1}, \infty ; s_{2}, \infty\right) ; M^{p_{1}, q_{2}}$ into $M\left(s_{1}, \infty ; t_{2}, \infty\right)$; $M^{q_{1}, p_{2}}$ into $M\left(t_{1}, \infty ; s_{2}, \infty\right)$ and $M^{q_{1}, q_{2}}$ into $M\left(t_{1}, \infty ; t_{2}, \infty\right)$, continuously. Then, interpolating we have

$$
\begin{aligned}
\mathbf{T}:\left(M^{p_{1}, p_{2}}, M^{p_{1}, q_{2}}\right)_{\theta, r_{2}} & \rightarrow\left(M\left(s_{1}, \infty ; s_{2}, \infty\right), M\left(s_{1}, \infty ; t_{2}, \infty\right)\right)_{\theta, r_{2}} \\
\mathbf{T}: M^{p_{1}, r_{2}} & \rightarrow M\left(s_{1}, \infty ; u_{2}, r_{2}\right)
\end{aligned}
$$

and

$$
\begin{aligned}
\mathbf{T}:\left(M^{q_{1}, p_{2}}, M^{q_{1}, q_{2}}\right)_{\theta, r_{2}} & \rightarrow\left(M\left(t_{1}, \infty ; s_{2}, \infty\right), M\left(t_{1}, \infty ; t_{2}, \infty\right)\right)_{\theta, r_{2}} \\
\mathbf{T}: M^{q_{1}, r_{2}} & \rightarrow M\left(t_{1}, \infty ; u_{2}, r_{2}\right) .
\end{aligned}
$$

Moreover, if $p_{2} \leq s_{2}$ and $q_{2} \leq t_{2}$, then $r_{2} \leq u_{2}$. Thus

$$
\begin{aligned}
\mathbf{T}:\left(M^{p_{1}, r_{2}}, M^{q_{1}, r_{2}}\right)_{\theta, r_{2}} \rightarrow\left(M\left(s_{1}, \infty ; u_{2}, r_{2}\right), M\left(t_{1}, \infty ; u_{2}, r_{2}\right)\right)_{\theta, r_{2}} \\
\mathbf{T}: M\left(r_{1}, r_{2} ; r_{2}, r_{2}\right) \rightarrow M\left(u_{1}, r_{2} ; u_{2}, u_{2}\right)
\end{aligned}
$$

continuously. 


\section{The space $A(P, Q, r)\left(\mathbb{R}^{d}\right)$}

Definition 18 Fix a non-zero window $g \in \mathcal{S}\left(\mathbb{R}^{d}\right), 1 \leq P=\left(p_{1}, p_{2}\right)<\infty, 1 \leq$ $Q=\left(q_{1}, q_{2}\right)<\infty$ and $1 \leq r<\infty$. The space $A(P, Q, r)\left(\mathbb{R}^{d}\right)$ is given by

$$
\begin{aligned}
A(P, Q, r)\left(\mathbb{R}^{d}\right) & =L^{r}\left(\mathbb{R}^{d}\right) \cap M(P, Q)\left(\mathbb{R}^{d}\right) \\
& =\left\{f \in L^{r}\left(\mathbb{R}^{d}\right) \mid V_{g} f \in L(P, Q)\left(\mathbb{R}^{2 d}\right)\right\} .
\end{aligned}
$$

It is easy to show that

$$
\|f\|_{A(P, Q, r)}=\|f\|_{r}+\|f\|_{M(P, Q)}=\|f\|_{r}+\left\|V_{g} f\right\|_{P Q}
$$

is a norm on the vector space $A(P, Q, r)\left(\mathbb{R}^{d}\right)$.

Theorem 19 Let $1<P<\infty, 1 \leq Q<\infty$, and $Q \leq P$, i.e. $q_{i} \leq p_{i}, i=1,2$. Then $\left(A(P, Q, r)\left(\mathbb{R}^{d}\right),\|.\|_{A(P, Q, r)}\right)$ is a Banach space.

Proof Let $\left(f_{n}\right)$ be a Cauchy sequence in $A(P, Q, r)\left(\mathbb{R}^{d}\right)$. Then $\left(f_{n}\right)$ and $\left(V_{g} f_{n}\right)$ are Cauchy sequence in $L^{r}\left(\mathbb{R}^{d}\right)$ and $L(P, Q)\left(\mathbb{R}^{2 d}\right)$, respectively. Since $L^{r}\left(\mathbb{R}^{d}\right)$ and $L(P, Q)\left(\mathbb{R}^{2 d}\right)$ are Banach spaces, there exists $f \in L^{r}\left(\mathbb{R}^{d}\right)$ and $h \in L(P, Q)\left(\mathbb{R}^{2 d}\right)$ such that

$$
\left\|f_{n}-f\right\|_{r} \rightarrow 0
$$

and

$$
\left\|V_{g} f_{n}-h\right\|_{P} \leq\left\|V_{g} f_{n}-h\right\|_{P Q} \rightarrow 0 .
$$

Thus $\left(V_{g} f_{n}\right)$ converges to the function $h$ in the mixed norm space $L^{P}\left(\mathbb{R}^{2 d}\right)=$ $L^{p_{1}, p_{2}}\left(\mathbb{R}^{2 d}\right)$. This implies that $\left(V_{g} f_{n}\right)$ has a subsequence $\left(V_{g} f_{n_{k}}\right)$ which converges pointwise to $h$ a.e. by the Theorem 1 [2, p. 304]. Moreover, since $f_{n}-f \in L^{r}\left(\mathbb{R}^{d}\right)$ and $M_{w} T_{x} g \in L^{r^{\prime}}\left(\mathbb{R}^{d}\right)$, using Hölder inequality and (3.1), we have

$$
\begin{aligned}
\left|V_{g} f_{n}(x, w)-V_{g} f(x, w)\right| & =\left|\left\langle f_{n}-f, M_{w} T_{x} g\right\rangle\right| \\
& \leq\left\|f_{n}-f\right\|_{r}\left\|M_{w} T_{x} g\right\|_{r^{\prime}} \\
& =\left\|f_{n}-f\right\|_{r}\|g\|_{r^{\prime}} \rightarrow 0 .
\end{aligned}
$$

Thus $\left(V_{g} f_{n}\right)$ converges pointwise to $V_{g} f$. Again, since $\left(f_{n}\right)$ is a Cauchy sequence, we get by (3.1)

$$
\begin{aligned}
\left|V_{g} f_{n_{k}}(x, w)-V_{g} f(x, w)\right| & \leq\left|\left\langle f_{n_{k}}-f_{n}, M_{w} T_{x} g\right\rangle\right|+\left|\left\langle f_{n}-f, M_{w} T_{x} g\right\rangle\right| \\
& \leq\left\|f_{n_{k}}-f_{n}\right\|_{r}\|g\|_{r^{\prime}}+\left\|f_{n}-f\right\|_{r}\|g\|_{r^{\prime}} \rightarrow 0 .
\end{aligned}
$$

Finally we obtain

$$
\left|V_{g} f(x, w)-h(x, w)\right| \leq\left|V_{g} f_{n_{k}}(x, w)-V_{g} f(x, w)\right|+\left|V_{g} f_{n_{k}}(x, w)-h(x, w)\right| \rightarrow 0
$$


and we have $V_{g} f=h$ a.e. Thus by (3.1) and (3.2), we have

$$
\begin{aligned}
\left\|f_{n}-f\right\|_{A(P, Q, r)} & =\left\|f_{n}-f\right\|_{r}+\left\|V_{g} f_{n}-V_{g} f\right\|_{P Q} \\
& =\left\|f_{n}-f\right\|_{r}+\left\|V_{g} f_{n}-h\right\|_{P Q} \rightarrow 0 .
\end{aligned}
$$

Hence $\left(A(P, Q, r)\left(\mathbb{R}^{d}\right),\|\cdot\|_{A(P, Q, r)}\right)$ is a Banach space.

Remark 20 Since $L^{r}\left(\mathbb{R}^{d}\right)$ and $M(P, Q)\left(\mathbb{R}^{d}\right)$ are invariant under time-frequency shifts for $1 \leq r<\infty, 1<P<\infty, 1 \leq Q<\infty$, then $A(P, Q, r)\left(\mathbb{R}^{d}\right)$ is invariant under time-frequency shifts. Again the function $(u, \eta) \longmapsto M_{\eta} T_{u} f$ is continuous on $\mathbb{R}^{2 d}$ into $L^{r}\left(\mathbb{R}^{d}\right)$ and on $\mathbb{R}^{2 d}$ into $M(P, Q)\left(\mathbb{R}^{d}\right)$, then it is also continuous on $\mathbb{R}^{2 d}$ into $A(P, Q, r)\left(\mathbb{R}^{d}\right)$. Moreover, as $L^{r}\left(\mathbb{R}^{d}\right)$ and $M(P, Q)\left(\mathbb{R}^{d}\right)$ are Banach convolution modules over $L^{1}\left(\mathbb{R}^{d}\right)$, then $A(P, Q, r)\left(\mathbb{R}^{d}\right)$ is a Banach convolution module over $L^{1}\left(\mathbb{R}^{d}\right)$, for $1<P<\infty, 1 \leq Q<\infty$ and $Q \leq P$. Also it can be shown that $A(P, Q, r)\left(\mathbb{R}^{d}\right)$ is an essential Banach module over $L^{1}\left(\mathbb{R}^{d}\right)$. The proof is the same as that in Theorem 2.2 in [16]. If $r=1,1<P<\infty, 1 \leq Q<\infty$ and $Q \leq P$, then we have

$$
\begin{aligned}
\|f * h\|_{A(P, Q, 1)} & =\|f * h\|_{1}+\|f * h\|_{M(P, Q)} \\
& \leq\|f\|_{1}\|h\|_{1}+\|f\|_{M(P, Q)}\|h\|_{1} \\
& =\|f\|_{A(P, Q, 1)}\|h\|_{1}
\end{aligned}
$$

for $f \in A(P, Q, 1)\left(\mathbb{R}^{d}\right)$ and $h \in L^{1}\left(\mathbb{R}^{d}\right)$. So $A(P, Q, 1)\left(\mathbb{R}^{d}\right)$ is a Banach ideal. Additionally, it is an essential Banach ideal over $L^{1}\left(\mathbb{R}^{d}\right)$ by module factorization theorem. Furthermore $A(P, Q, 1)\left(\mathbb{R}^{d}\right)$ is a Banach convolution algebra for $r=1,1<$ $P<\infty, 1 \leq Q<\infty$ and $Q \leq P$.

Theorem 21 If $r=1,1<P<\infty, 1 \leq Q<\infty$ and $Q \leq P$, then the space $A(P, Q, 1)\left(\mathbb{R}^{d}\right)$ is a Segal algebra.

Proof It is known that from the Remark 20, the space $A(P, Q, 1)\left(\mathbb{R}^{d}\right)$ is a Banach convolution algebra, strongly translation invariant and translation operator is continuous on $\mathbb{R}^{d}$ into $A(P, Q, 1)\left(\mathbb{R}^{d}\right)$. For the proof it is enough to see that $A(P, Q, 1)\left(\mathbb{R}^{d}\right)$ is dense in $L^{1}\left(\mathbb{R}^{d}\right)$. By Proposition 2, we have $\mathcal{S}\left(\mathbb{R}^{d}\right) \subset M(P, Q)\left(\mathbb{R}^{d}\right)$. It is also known that $\mathcal{S}\left(\mathbb{R}^{d}\right) \subset L^{1}\left(\mathbb{R}^{d}\right)$ is dense in $L^{1}\left(\mathbb{R}^{d}\right)$. As $\mathcal{S}\left(\mathbb{R}^{d}\right) \subset L^{1}\left(\mathbb{R}^{d}\right) \subset A(P, Q, 1)\left(\mathbb{R}^{d}\right)$ and $\mathcal{S}\left(\mathbb{R}^{d}\right)$ is dense in $L^{1}\left(\mathbb{R}^{d}\right)$, then $A(P, Q, 1)\left(\mathbb{R}^{d}\right)$ is dense in $L^{1}\left(\mathbb{R}^{d}\right)$.

For $1<P<\infty, 1 \leq Q<\infty$, let the function

$$
\Phi: A(P, Q, r)\left(\mathbb{R}^{d}\right) \rightarrow L^{r}\left(\mathbb{R}^{d}\right) \times L(P, Q)\left(\mathbb{R}^{2 d}\right)
$$

be given by $\Phi(f)=\left(f, V_{g} f\right)$. Also let

$$
H=\left\{\left(f, V_{g} f\right): f \in A(P, Q, r)\left(\mathbb{R}^{d}\right)\right\}=\Phi\left(A(P, Q, r)\left(\mathbb{R}^{d}\right)\right) .
$$

Then

$$
\|\Phi(f)\|=\left\|\left(f, V_{g} f\right)\right\|=\|f\|_{r}+\left\|V_{g} f\right\|_{P Q}
$$


is a norm on $H$. Thus $\Phi$ is an isometry. Moreover we set

$$
K=\left\{\begin{array}{c}
(\varphi, \psi):(\varphi, \psi) \in L^{r^{\prime}}\left(\mathbb{R}^{d}\right) \times L\left(P^{\prime}, Q^{\prime}\right)\left(\mathbb{R}^{2 d}\right), \\
\int_{\mathbb{R}^{d}} f(y) \varphi(y) d y+\iint_{\mathbb{R}^{2 d}} V_{g} f(z) \psi(z) d z=0, \text { for all }\left(f, V_{g} f\right) \in H
\end{array}\right\}
$$

where $\frac{1}{P}+\frac{1}{P^{\prime}}=1, \frac{1}{Q}+\frac{1}{Q^{\prime}}=1$ and $\frac{1}{r}+\frac{1}{r^{\prime}}=1$.

Proposition 22 Let $1<P<\infty, 1 \leq Q<\infty$. Then the dual space of the space $A(P, Q, r)\left(\mathbb{R}^{d}\right)$ is isomorphic to $L^{r^{\prime}}\left(\mathbb{R}^{d}\right) \times L\left(P^{\prime}, Q^{\prime}\right)\left(\mathbb{R}^{2 d}\right) / K$, where $\frac{1}{P}+\frac{1}{P^{\prime}}=1$, $\frac{1}{Q}+\frac{1}{Q^{\prime}}=1$ and $\frac{1}{r}+\frac{1}{r^{\prime}}=1$.

Proof This proposition is easily proved by the Duality Theorem 1.7 in [19].

\section{Multiplier spaces}

\subsection{Multiplier spaces of $\mathrm{M}(P, Q)\left(\mathbb{R}^{d}\right)$}

Proposition 23 Let $1<P<\infty, 1 \leq Q<\infty$. If $\frac{1}{P}+\frac{1}{P^{\prime}}=1$ and $\frac{1}{Q}+\frac{1}{Q^{\prime}}=1$, then

1. $\operatorname{Hom}_{L^{1}\left(\mathbb{R}^{d}\right)}\left(L^{1}\left(\mathbb{R}^{d}\right), M\left(P^{\prime}, Q^{\prime}\right)\left(\mathbb{R}^{d}\right)\right)=M\left(P^{\prime}, Q^{\prime}\right)\left(\mathbb{R}^{d}\right)$.

2. $\operatorname{Hom}_{L^{1}}\left(M(P, Q)\left(\mathbb{R}^{d}\right), L^{\infty}\left(\mathbb{R}^{d}\right)\right)=\operatorname{Hom}_{L^{1}}\left(L^{1}\left(\mathbb{R}^{d}\right), M\left(P^{\prime}, Q^{\prime}\right)\left(\mathbb{R}^{d}\right)\right)$.

Proof 1. Using Theorem 8, Theorem 13 and [22, Corollary 2.13], we obtain

$$
\begin{aligned}
\operatorname{Hom}_{L^{1}\left(\mathbb{R}^{d}\right)}\left(L^{1}\left(\mathbb{R}^{d}\right), M\left(P^{\prime}, Q^{\prime}\right)\left(\mathbb{R}^{d}\right)\right) & =\left(L^{1}\left(\mathbb{R}^{d}\right) * M(P, Q)\left(\mathbb{R}^{d}\right)\right)^{*} \\
& =\left(M(P, Q)\left(\mathbb{R}^{d}\right)\right)^{*}=M\left(P^{\prime}, Q^{\prime}\right)\left(\mathbb{R}^{d}\right) .
\end{aligned}
$$

2. It is known that the dual space of $L^{1}\left(\mathbb{R}^{d}\right)$ is $L^{\infty}\left(\mathbb{R}^{d}\right)$. Applying again [22, Corollary 2.13] we have

$$
\begin{aligned}
\operatorname{Hom}_{L^{1}}\left(M(P, Q)\left(\mathbb{R}^{d}\right), L^{\infty}\left(\mathbb{R}^{d}\right)\right) & =\left(L^{1}\left(\mathbb{R}^{d}\right) * M(P, Q)\left(\mathbb{R}^{d}\right)\right)^{*} \\
& =\left(M(P, Q)\left(\mathbb{R}^{d}\right)\right)^{*}=M\left(P^{\prime}, Q^{\prime}\right)\left(\mathbb{R}^{d}\right) .
\end{aligned}
$$

Corollary 24 If we take $p_{1}=q_{1}=p$ and $p_{2}=q_{2}=q$ in Proposition 23, then we obtain

1. $\operatorname{Hom}_{L^{1}\left(\mathbb{R}^{d}\right)}\left(L^{1}\left(\mathbb{R}^{d}\right), M^{p^{\prime}, q^{\prime}}\left(\mathbb{R}^{d}\right)\right)=M^{p^{\prime}, q^{\prime}}\left(\mathbb{R}^{d}\right)$.

2. $\operatorname{Hom}_{L^{1}}\left(M^{p, q}\left(\mathbb{R}^{d}\right), L^{\infty}\left(\mathbb{R}^{d}\right)\right)=\operatorname{Hom}_{L^{1}}\left(L^{1}\left(\mathbb{R}^{d}\right), M^{p^{\prime}, q^{\prime}}\left(\mathbb{R}^{d}\right)\right)$, where $M^{p, q}\left(\mathbb{R}^{d}\right)$ and $M^{p^{\prime}, q^{\prime}}\left(\mathbb{R}^{d}\right)$ are the standard modulation spaces.

\subsection{Multiplier spaces of $\mathrm{A}(P, Q, r)\left(\mathbb{R}^{d}\right)$}

Consider a net $\left(e_{\alpha}\right)$, which is a bounded approximate identity in $L^{1}\left(\mathbb{R}^{d}\right)$ and having $\hat{e_{\alpha}}$ with compact support, $\forall \alpha \in I$. Define

$$
M_{A(P, Q, 1)}\left(\mathbb{R}^{d}\right)=\left\{\mu \in M\left(\mathbb{R}^{d}\right) \mid\left\|\mu * e_{\alpha}\right\|_{A(P, Q, 1)} \leq C(\mu)\right\},
$$


where

$$
\|\mu\|_{M_{A(P, Q, 1)}}=\sup \left\{\frac{\left\|\mu * e_{\alpha}\right\|_{A(P, Q, 1)}}{\left\|e_{\alpha}\right\|_{1}}\right\}
$$

$M\left(\mathbb{R}^{d}\right)$ is the space of bounded regular Borel measure on $\mathbb{R}^{d}$ and $C(\mu)$ is a constant depending on the measure $\mu$. As $A(P, Q, 1)\left(\mathbb{R}^{d}\right)$ is a Segal algebra and an essential Banach ideal for $1<P<\infty, 1 \leq Q<\infty$ and $Q \leq P$, then $M_{A(P, Q, 1)}\left(\mathbb{R}^{d}\right)$ is uniquely defined as independent of the approximate identity, for $1<P<\infty, 1 \leq$ $Q<\infty$ and $Q \leq P$ by Proposition 3 in [10].

Proposition 25 Let $1<P<\infty, 1 \leq Q<\infty$ and $Q \leq P$. Then for a linear operator $T: L^{1}\left(\mathbb{R}^{d}\right) \rightarrow A(P, Q, 1)\left(\mathbb{R}^{\bar{d}}\right)$, the following are equivalent:

1. $T \in M\left(L^{1}\left(\mathbb{R}^{d}\right), A(P, Q, 1)\left(\mathbb{R}^{d}\right)\right)$.

2. There exists a unique measure $\mu \in M_{A(P, Q, 1)}\left(\mathbb{R}^{d}\right)$ such that $T f=\mu * f$ for every $f \in L^{1}\left(\mathbb{R}^{d}\right)$. Moreover, the spaces $M\left(L^{1}\left(\mathbb{R}^{d}\right), A(P, Q, 1)\left(\mathbb{R}^{d}\right)\right)$ and $M_{A(P, Q, 1)}\left(\mathbb{R}^{d}\right)$ are homeomorphic.

Proof If $1<P<\infty, 1 \leq Q<\infty$ and $Q \leq P$, it is known that the space $A(P, Q, 1)\left(\mathbb{R}^{d}\right)$ is a Segal algebra and an essential Banach ideal by Theorem 21 and Remark 20. Thus by Theorem 4 in [10], the proof is completed.

The next proposition is proved as Proposition 23.

Proposition 26 Let $1<P<\infty, 1 \leq Q<\infty$ and $Q \leq P$. Then $\operatorname{Hom}_{L^{1}}\left(A(P, Q, r)\left(\mathbb{R}^{d}\right), L^{\infty}\left(\mathbb{R}^{d}\right)\right)$ and $\operatorname{Hom}_{L^{1}}\left(L^{1}\left(\mathbb{R}^{d}\right),\left(A(P, Q, r)\left(\mathbb{R}^{d}\right)\right)^{*}\right)$ are isomorphic to $L^{r^{\prime}}\left(\mathbb{R}^{d}\right) \times L\left(P^{\prime}, Q^{\prime}\right)\left(\mathbb{R}^{2 d}\right) / K$, where $\frac{1}{P}+\frac{1}{P^{\prime}}=1, \frac{1}{Q}+\frac{1}{Q^{\prime}}=1$ and $\frac{1}{r}+\frac{1}{r^{\prime}}=1$ and $\left(A(P, Q, r)\left(\mathbb{R}^{d}\right)\right)^{*}$ is the dual of $A(P, Q, r)\left(\mathbb{R}^{d}\right)$.

Theorem 27 Let $1<P, Q<\infty, Q \leq P, g \in \mathcal{S}\left(\mathbb{R}^{d}\right) \backslash\{0\}$ and $T: A(P, Q, 1)$ $\left(\mathbb{R}^{d}\right) \rightarrow L^{1}\left(\mathbb{R}^{d}\right)$ be a linear transformation. Then the following are equivalent:

1. $T \in M\left(A(P, Q, 1)\left(\mathbb{R}^{d}\right), L^{1}\left(\mathbb{R}^{d}\right)\right)$.

2. There exists a unique $\mu \in M\left(\mathbb{R}^{d}\right)$ such that $T f=\mu * f$ for every $f \in$ $A(P, Q, 1)\left(\mathbb{R}^{d}\right)$, where $M\left(\mathbb{R}^{d}\right)$ is the space of bounded regular Borel measure on $\mathbb{R}^{d}$.

Proof Let $\mu \in M\left(\mathbb{R}^{d}\right)$ and $T f=\mu * f$ for every $f \in A(P, Q, 1)\left(\mathbb{R}^{d}\right)$. Hence we get

$$
\|T f\|_{1}=\|\mu * f\|_{1} \leq\|\mu\|\|f\|_{1} \leq\|\mu\|\|f\|_{A(P, Q, 1)} .
$$

It is easy to see the other conditions to be multiplier from $A(P, Q, 1)\left(\mathbb{R}^{d}\right)$ into $L^{1}\left(\mathbb{R}^{d}\right)$. Thus $T \in M\left(A(P, Q, 1)\left(\mathbb{R}^{d}\right), L^{1}\left(\mathbb{R}^{d}\right)\right)$. Conversely, let $T \in$ $M\left(A(P, Q, 1)\left(\mathbb{R}^{d}\right), L^{1}\left(\mathbb{R}^{d}\right)\right)$. Thus we have

$$
\|T f\|_{1} \leq\|T\|\|f\|_{A(P, Q, 1)}=\|T\|\left(\|f\|_{1}+\left\|V_{g} f\right\|_{P Q}\right) .
$$


By (4.1) and the inequality (4.10) in [16], we have

$$
2\|T f\|_{1} \leq\|T\|\left(2\|f\|_{1}+\lim _{s \rightarrow \infty}\left\|V_{g} f+e^{-2 \pi i s w} T_{(s, 0)} V_{g} f\right\|_{P Q}\right)
$$

for all $f \in A(P, Q, 1)\left(\mathbb{R}^{d}\right)$. Again by using Lemma 4.1 and Lemma 4.2 in [16], we have

$\lim _{s \rightarrow \infty}\left\|V_{g} f+e^{-2 \pi i s w} T_{(s, 0)} V_{g} f\right\|_{p_{1} q_{1}}=\lim _{s \rightarrow \infty}\left\|V_{g} f+T_{(s, 0)} V_{g} f\right\|_{p_{1} q_{1}}=2^{\frac{1}{p_{1}}}\left\|V_{g} f\right\|_{p_{1} q_{1}}$.

The continuity of $\|\cdot\|_{p_{2} q_{2}}$ implies that

$$
\left\|V_{g} f+e^{-2 \pi i s w} T_{(s, 0)} V_{g} f\right\|_{P Q} \rightarrow 2^{\frac{1}{p_{1}}}\left\|V_{g} f\right\|_{P Q}
$$

as $s \rightarrow \infty$. Hence we obtain

$$
2\|T f\|_{1} \leq\|T\|\left(2\|f\|_{1}+2^{\frac{1}{p_{1}}}\left\|V_{g} f\right\|_{P Q}\right)
$$

and

$$
\|T f\|_{1} \leq\|T\|\left(\|f\|_{1}+2^{\frac{1}{p_{1}}-1}\left\|V_{g} f\right\|_{P Q}\right)
$$

Repeating this process $n$ times, we have

$$
\|T f\|_{1} \leq\|T\|\left(\|f\|_{1}+2^{n\left(\frac{1}{p_{1}}-1\right)}\left\|V_{g} f\right\|_{P Q}\right)
$$

for all $f \in A(P, Q, 1)\left(\mathbb{R}^{d}\right)$. Since $p_{1}>1$ we have $\lim _{n \rightarrow \infty} 2^{n\left(\frac{1}{p_{1}}-1\right)}=0$, thus we get through

$$
\|T f\|_{1} \leq\|T\|\|f\|_{1}
$$

So, since $T$ is a continuous linear transformation from $A(P, Q, 1)\left(\mathbb{R}^{d}\right)$ to $L^{1}\left(\mathbb{R}^{d}\right)$ and $A(P, Q, 1)\left(\mathbb{R}^{d}\right)$ is dense in $L^{1}\left(\mathbb{R}^{d}\right)$, then $T$ has a unique continuous linear extension $\widetilde{T}: L^{1}\left(\mathbb{R}^{d}\right) \rightarrow L^{1}\left(\mathbb{R}^{d}\right)$ and $\|\widetilde{T}\|=\|T\|$. Thus there exists a unique measure $\mu \in M\left(\mathbb{R}^{d}\right)$ such that $T f=\mu * f$ for all $f \in A(P, Q, 1)\left(\mathbb{R}^{d}\right)$ by Theorem 0.1.1 in [18].

The following theorem can be easily proved by Theorem 4.2 in [16].

Theorem 28 If $1<P, Q<\infty$ and $Q \leq P$, then the multipliers $M\left(A(P, Q, 1)\left(\mathbb{R}^{d}\right)\right.$, $\left.A(P, Q, 1)\left(\mathbb{R}^{d}\right)\right)$ is isometrically isomorphic to $M\left(\mathbb{R}^{d}\right)$.

Acknowledgments The author would like to thank Elena Cordero, Luigi Rodino, Joachim Toft, M. W. Wong and Patrik Wahlberg for fruitful discussions and comments. This work was started while she was visiting the Department of Mathematics of the University of Torino. She also wants to thank Elena Cordero and Luigi Rodino for their wonderful hospitality and for great working conditions. 
Open Access This article is distributed under the terms of the Creative Commons Attribution License which permits any use, distribution, and reproduction in any medium, provided the original author(s) and the source are credited.

\section{References}

1. Bastero, J., Milman, M., Ruiz, F.: A note on $L(\infty, q)$ spaces and Sobolev embeddings, vol. 5. Indiana Univ. Math. J. 52, 1215-1230 (2003)

2. Benedek, A., Panzone, R.: The spaces $L^{P}$, with mixed norm. Duke Math. J. 28, 301-324 (1961)

3. Bennet, C., DeVore, R.A., Sharpley, R.: Weak- $L^{\infty}$ and BMO. vol. 3. Ann. Math. 113, 601-611 (1981)

4. Bennet, C., Sharpley, R.: Interpolation of Operators. Academic Press Inc., San diego (1998)

5. Blozinski, A.P.: On a convolution theorem for $L(p, q)$ spaces. Trans. Am. Math. Soc. 164, 255-265 (1972)

6. Blozinski, A.P.: Multivariate rearrangements and Banach function spaces with mixed norms, vol. 1. Trans. Am. Math. Soc. 263, 149-167 (1981)

7. Chen, Y.K., Lai, H.C.: Multipliers of Lorentz spaces. Hokkaido Math. J. 4, 247-260 (1975)

8. Cordero, E, Gröchenig, K.: Time-frequency analysis of localization operators. J. Funct. Anal. 205, 107-131 (2003)

9. Cwikel, M.: On $\left(L^{p_{0}}\left(A_{0}\right), L^{p_{1}}\left(A_{1}\right)\right)_{\theta, q}$, vol. 2. Proc. Am. Math. Soc. 44, 286-292 (1974)

10. Doğan, M, Gürkanlı, A.T.: Multipliers of the space $S_{\omega}(G)$. Math. Balkanica. New Series 15(3-4), 199-212 (2001)

11. Feichtinger, H.G.: Modulation spaces on locally compact Abelian groups. Technical report, University of Vienna (1983)

12. Feichtinger, H.G., Gürkanlı, A.T.: On a family of weighted convolution algebras, vol. 3. Internat J. Math. Math. Sci. 13, 517-526 (1990)

13. Fernandez, D.L.: Lorentz spaces, with mixed norms. J. Funct. Anal. 25, 128-146 (1977)

14. Gröchenig, K.: Foundations of Time-Frequency Analysis. Birkhäuser, Boston (2001)

15. Gürkanlı, A.T.: Multipliers of some Banach ideals and Wiener-Ditkin sets, vol. 2. Math. Slovaca. 55, 237-248 (2005)

16. Gürkanlı, A.T.: Time frequency analysis and multipliers of the spaces $M(p, q)\left(\mathbb{R}^{d}\right)$ and $S(p, q)\left(\mathbb{R}^{d}\right)$, vol. 3. J. Math. Kyoto Univ. 46, 595-616 (2006)

17. Hunt, R.A.: On $L(p, q)$ Spaces, vol. 4. Extrait de L'Enseignement Mathematique 12, 249-276 (1996)

18. Larsen, R.: Introduction to the Theory of Multipliers. Springer, Berlin (1971)

19. Liu, T.S., Van Rooij, A.: Sums and intersections of normed linear spaces. Math. Nachr. 42, 29-42 (1969)

20. Milman, M.: On interpolation of $2^{n}$ Banach spaces and Lorentz spaces with mixed norms. J. Funct. Anal. 41, 1-7 (1981)

21. O'Neil, R.: Convolution operators and $L(p, q)$ spaces. Duke Math. J. 30, 129-142 (1963)

22. Rieffel, M.A.: Induced Banach representation of Banach algebras and locally compact groups. J. Funct. Anal. 1, 443-491 (1967)

23. Rieffel, M.A.: Multipliers and tensor products of $L^{p}$-spaces of locally compact groups. Studia Math. 33, 71-82 (1969)

24. Sandıkçı, A., Gürkanlı, A.T.: Gabor Analysis of the spaces $M(p, q, w)\left(\mathbb{R}^{d}\right)$ and $S(p, q, r, w, \omega)\left(\mathbb{R}^{d}\right)$, vol. 1. Acta Mathematica Scientia. 31B, 141-158 (2011) 